\title{
Monte-Carlo modelling to determine optimum filter choices for sub-microsecond optical pyrometry
}

Thomas A. Ota, David J. Chapman, and Daniel E. Eakins

Citation: Review of Scientific Instruments 88, 044902 (2017); doi: 10.1063/1.4978906

View online: https://doi.org/10.1063/1.4978906

View Table of Contents: http://aip.scitation.org/toc/rsi/88/4

Published by the American Institute of Physics

\section{Articles you may be interested in}

Determination of the resistivity anisotropy of orthorhombic materials via transverse resistivity measurements

Review of Scientific Instruments 88, 043901 (2017); 10.1063/1.4978908

A direct differential method for measuring thermal conductivity of thin films

Review of Scientific Instruments 88, 044901 (2017); 10.1063/1.4979163

Nonmechanical compact probe for cross-sectional velocity measurement based on differential laser Doppler velocimetry

Review of Scientific Instruments 88, 045001 (2017); 10.1063/1.4979563

Fast, precise, and widely tunable frequency control of an optical parametric oscillator referenced to a frequency comb

Review of Scientific Instruments 88, 033101 (2017); 10.1063/1.4977049

Invited Review Article: Tip modification methods for tip-enhanced Raman spectroscopy (TERS) and colloidal probe technique: A 10 year update (2006-2016) review

Review of Scientific Instruments 88, 031101 (2017); 10.1063/1.4978929

Absolute optical responsivity down to the photon counting level with a photomultiplier tube

Review of Scientific Instruments 88, 043104 (2017); 10.1063/1.4979812

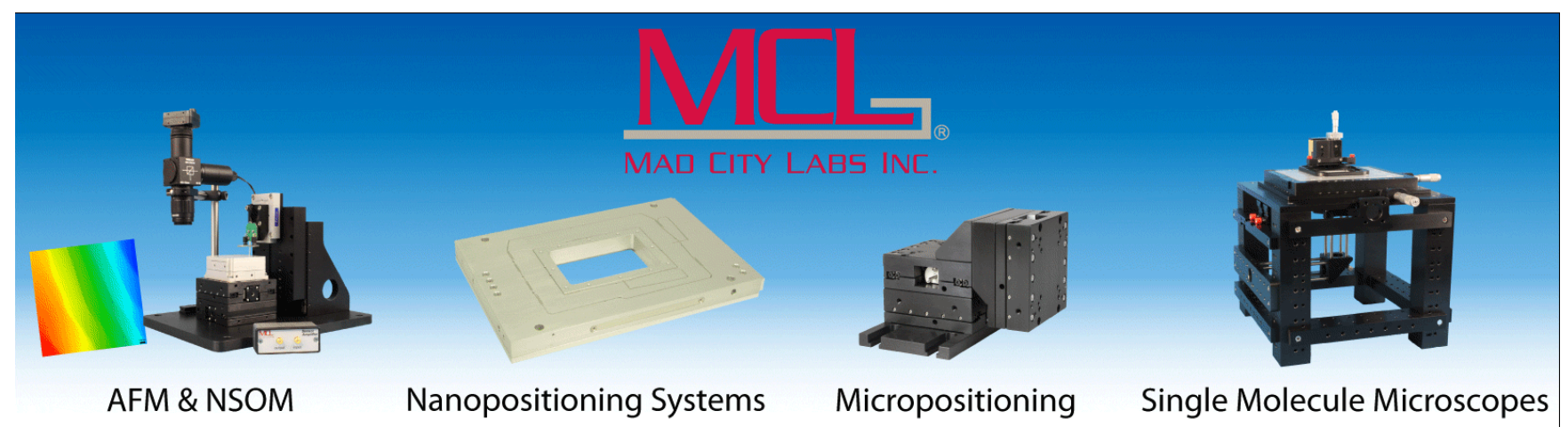




\title{
Monte-Carlo modelling to determine optimum filter choices for sub-microsecond optical pyrometry
}

\author{
Thomas A. Ota, ${ }^{1,2, a)}$ David J. Chapman, ${ }^{2}$ and Daniel E. Eakins ${ }^{2}$ \\ ${ }^{1} A W E$, Aldermaston, Reading, Berkshire RG7 4PR, United Kingdom \\ ${ }^{2}$ Institute of Shock Physics, Blackett Laboratory, Imperial College London, London, United Kingdom
}

(Received 6 September 2016; accepted 5 March 2017; published online 4 April 2017)

\begin{abstract}
When designing a spectral-band pyrometer for use at high time resolutions (sub- $\mu \mathrm{s}$ ), there is ambiguity regarding the optimum characteristics for a spectral filter(s). In particular, while prior work has discussed uncertainties in spectral-band pyrometry, there has been little discussion of the effects of noise which is an important consideration in time-resolved, high speed experiments. Using a MonteCarlo process to simulate the effects of noise, a model of collection from a black body has been developed to give insights into the optimum choices for centre wavelength and passband width. The model was validated and then used to explore the effects of centre wavelength and passband width on measurement uncertainty. This reveals a transition centre wavelength below which uncertainties in calculated temperature are high. To further investigate system performance, simultaneous variation of the centre wavelength and bandpass width of a filter is investigated. Using data reduction, the effects of temperature and noise levels are illustrated and an empirical approximation is determined. The results presented show that filter choice can significantly affect instrument performance and, while best practice requires detailed modelling to achieve optimal performance, the expression presented can be used to aid filter selection. [http://dx.doi.org/10.1063/1.4978906]
\end{abstract}

\section{INTRODUCTION}

High strain rate experiments offer the ability to access states of matter not readily achievable by other methods. For this reason, such experiments are routinely performed to develop equation-of-state (EoS) models which describe the relationship between a material's state variables over a wide range of thermodynamic states. Historically, development of EoS models has been largely based on plate impact experiments where the pressure and density state variables, which can be deduced from measurements of shock and particle velocities using the Rankine-Hugoniot relations, are determined. Additional state variables are often calculated based on assumptions about the material and thus result in large uncertainties. Improving the accuracy of EoS models requires measurement of a third state variable. Measurement of temperature can be achieved using optical pyrometry, an established method to measure temperature in high strain rate experiments. ${ }^{1-3}$

In optical pyrometry, temperature is inferred by measuring the light emitted by a sample due to thermal emission over one, or more, spectral bands. There are two potentially significant sources of uncertainty in this process: sample emissivity and the measurement of radiance. Emissivity can vary with state variables; if emissivity is not measured, significant uncertainty can result. ${ }^{4,5}$ Progress in the measurement of reflectivity (and hence its complement emissivity) has been reported. ${ }^{3,6,7}$ However, further understanding of the uncertainty in a radiance measurement is also desirable as the resultant uncertainty in temperature is a combination of both uncertainties. The

a) thomas.ota10@imperial.ac.uk discussion here focuses on the uncertainties in a thermal radiance measurement.

Despite the conceptual simplicity of a spectral band pyrometer, there are numerous considerations when configuring the system, these include pyrometer topology, lens selection, detector choices, and filter parameters. Consideration of the number of permutations of detectors, filters, and lenses indicates it is not feasible to determine the optimum pyrometer experimentally. Furthermore, the increased complexity of an experimental system can obfuscate the effect of changing one component. For example, increasing the centre wavelength of a filter may decrease signal levels due to detector response, despite the increased emittance of a thermal source at longer wavelengths.

Complexities arise even if considerations are limited to which spectral bands to use. For example, moving the wavelength band toward the peak in the emission spectrum will make more light available for detection. However, it has been previously demonstrated that collection of light at shorter wavelengths allows the temperature to be determined more accurately as, in this section of the spectrum, small changes in temperature result in large changes in spectral radiance. ${ }^{8}$ Similarly, it is clear that by increasing the passband of a filter more light will be available to be detected; however, it is not clear that this increase will reduce the uncertainty in the temperature measurement. The conflicting guidance of these heuristics indicates a complexity that requires deeper investigation to resolve.

Thus a simplified model is needed to investigate the effects of changing individual components to identify the configuration changes that most significantly reduce measurement uncertainty. In this paper, we focus on the combined effects 
of the wavelengths and wavelength ranges at which measurements will be taken as these are a fundamental choice when configuring a spectral-band pyrometer. Even restricted to these two variables, it is not trivial to identify the most appropriate choices, and indeed this is a long-standing challenge. ${ }^{9-11}$ While the published literature provides useful insights, they overlook the requirement for high temporal resolution and are thus not immediately relevant to high strain rate experiments. In particular, although high strain rate experiments allow extreme states to be reached, these states are only maintained for a short time. Considering plate impact experiments in particular, well defined states are maintained for $\sim \mu$ s requiring sub- $\mu$ s time resolution diagnostics. Correspondingly the high bandwidths required mean it is necessary to consider the effects of noise as this can significantly influence which wavelength ranges are most appropriate.

\section{DEVELOPMENT OF A MONTE-CARLO MODEL}

\section{A. Calculation of radiance}

An idealised model has been developed to attempt to develop a quantitative understanding of the aforementioned compromises. The model consists of a source, a filter, and a detector. Some limitations on the system being considered have been set. First, due to the availability of other techniques at higher temperatures, the issue regarding filter wavelength and passband is particularly relevant at low temperatures where spectral-band pyrometry is the most viable technique. As such it shall be assumed that the temperature will be less than $2000 \mathrm{~K}$.

The transmission of the filter, $T(\lambda)$, was parameterised by a centre wavelength, $\lambda_{c}$, and passband $\Delta \lambda$. It was also assumed that the filters used are "ideal" in that they have $100 \%$ transmission in the passband and $0 \%$ transmission out of band, as specified by the following equation:

$$
T(\lambda)= \begin{cases}0, & \left|\lambda-\lambda_{c}\right|>\frac{\Delta \lambda}{2} \\ 1, & \left|\lambda-\lambda_{c}\right| \leq \frac{\Delta \lambda}{2}\end{cases}
$$

In addition, shock experiments require high speed detectors; such detectors operate predominately in the visible and near-infrared part of the electromagnetic (EM) spectrum. Furthermore, many high speed pyrometry measurements use silica optical fibre, which has high propagation losses at longer wavelengths. Thus a second limitation was that only centre wavelengths from $300 \mathrm{~nm}$ to $2000 \mathrm{~nm}$ will be considered.

Finally, to remove the additional uncertainties related to material specific emissivity, it was assumed that these measurements are to be made on a blackbody (i.e., emissivity of one at all wavelengths). Thus the radiant output of the source is given by Planck's law

$$
L_{\lambda}(\lambda, T)=\frac{2 h c_{0}^{2}}{\lambda^{5}} \frac{1}{e^{\frac{h c}{\lambda k_{B} T}}-1},
$$

where $L_{\lambda}$ is the spectral radiance in $\mathrm{W} \mathrm{sr}^{-1} \mathrm{~m}^{-3}, h$ is Planck's constant, $c_{0}$ is the speed of light in a vacuum, $\lambda$ is wavelength, $k_{B}$ is Boltzmann's constant, and $T$ is temperature of the source.
Monte-Carlo simulations have been performed to investigate the uncertainties of the process of converting radiance to temperatures. The approach proceeds as follows:

1. Planck's law, Equation (2), is used to calculate the radiant output of a blackbody emitter viewed by an optical system with a filter specified by $\lambda_{c}$ and $\Delta \lambda$ at a range of temperatures.

2. The detection of the light is simulated assuming a detector with an ideal spectral response of $1 \mathrm{~V} \mathrm{~W}^{-1}$ for all wavelengths. Noise is generated via a Monte-Carlo process and added to the signal to simulate the uncertainties inherent in performing a high speed measurement. The probability distribution of the noise is determined by an appropriate noise model, discussed in Section II B.

3. The previously calculated radiance-temperature relation is used to convert the simulated measurement into a temperature. This process is repeated until a statistically reliable value of the uncertainty of the process, parameterised by the standard deviation, is found.

4. The properties (i.e., $\lambda_{c}$ or $\Delta \lambda$ or both) of the filter are changed and the process is repeated.

To calculate the energy output from a blackbody over a specific wavelength band, it is necessary to integrate Planck's law over the wavelength range of interest and scale for optical coupling effects. To convert measured radiance, $R$, to a temperature, $T$, the inverse process is required but there is no analytical solution for this process. Thus it was necessary to calculate a curve for $R(T, \lambda)$ then invert it numerically to calculate $T(R, \lambda)$. Figure 1 shows curves created by this process for a number of filter parameters. It can be seen that in all cases, the gradient of this curve is steeper at lower temperatures making the measurement more sensitive. However this is balanced by the correspondingly lower signal levels that mean any measurements in this region are likely to have a larger proportional uncertainty (assuming constant noise). As the accuracy of the measurement is dependent on the noise of the measurement, it is necessary to consider an appropriate noise model.

\section{B. Noise model}

The noise model proposed has three components: shot noise, detector noise, and digitiser noise. Shot noise is due to

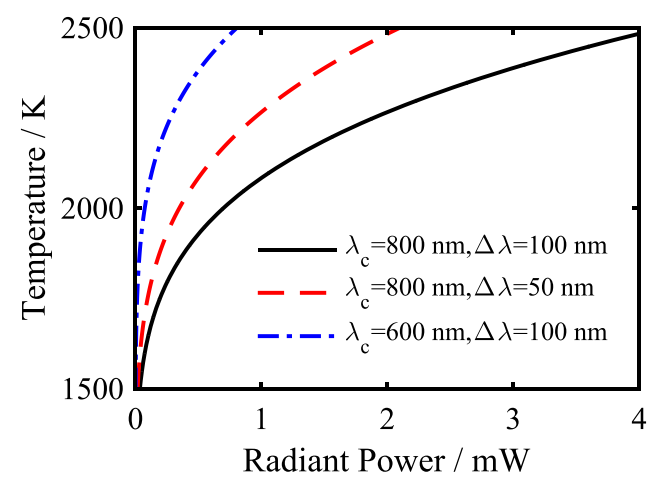

FIG. 1. A radiance-temperature curve showing how the radiance of a blackbody can be related to its temperature over three filter passbands. The legend indicates the filter parameters. 
the quantised nature of light and is a function of the number of photons collected. This noise is assumed to have a Gaussian probability distribution, $p_{G}$, given by

$$
p_{G}(z)=\frac{1}{\sigma_{\text {shot }} \sqrt{2 \pi}} e^{-\frac{z^{2}}{2 \sigma_{\text {shot }}^{2}}},
$$

where the standard deviation of the distribution, $\sigma_{\text {shot }}$, is given by

$$
\sigma_{\text {shot }}=\sqrt{\frac{P_{o p t}}{h v_{c} B}} .
$$

The number of photons was calculated using the calculated optical power, $P_{\text {opt }}$; the energy per photon, which assuming the photons are the centre frequency of the filter, is given by $h v_{c}$, and the time interval, which is related to the bandwidth $B$ of the recording system.

Detector noise is a description of the noise inherent in all optical detectors (although not necessarily from the same source) and is specified by the noise equivalent power (NEP) and the bandwidth, $B$. Like shot noise, detector noise has a Gaussian distribution and is parameterised by its standard deviation $\sigma_{\text {det }}$,

$$
\sigma_{d e t}=\operatorname{NEP} \sqrt{B}
$$

Digitiser noise is the noise due to the digital acquisition of the signal and can be approximated as a fixed percentage of the signal. It has two components, quantisation noise and dithering. Quantisation noise is due to the quantisation process which implicitly adds noise. ${ }^{12}$ Dithering is noise that is purposely added by digitiser manufacturers to improve resolution when over-sampling. ${ }^{13}$ Both are assumed to have a Gaussian distribution and are parameterised by their standard deviations, $\sigma_{q n t}$ for quantisation noise and $\sigma_{d t h}$ for dithering. The expressions for the standard deviations are

$$
\sigma_{q n t}=\frac{1}{\sqrt{12}} 2^{-Q}
$$

and

$$
\sigma_{d t h}=\frac{2}{3} 2^{-Q},
$$

where because the noise is related to the minimum resolvable voltage (least significant bit), $Q$ is the bit depth of the digitiser. It is assumed the signal scales to the digitiser input range, thus the previous values are fractions of the recorded value. They can be combined in a single term, $\sigma_{\text {dig }}$, given by

$$
\sigma_{d i g}=P_{o p t} \sqrt{\sigma_{q n t}^{2}+\sigma_{d t h}^{2}} .
$$

As these three components of noise are uncorrelated, they are added in quadrature to determine the resultant noise on the signal

$$
\sigma=\sqrt{\sigma_{\text {shot }}^{2}+\sigma_{d e t}^{2}+\sigma_{d i g}^{2}}
$$

\section{MODEL VALIDATION EXPERIMENTS}

It is desirable to experimentally validate the approach before using it to systematically investigate system behaviour. To achieve this, a calibrated voltage-temperature curve, $V(T)$, is required to allow measured radiances to be converted to temperature. The Monte-Carlo model does not produce such a curve due to the noise that is added. Even if the noise component was removed, there would be numerous inaccuracies, in comparison to experimental systems, due to the ideal components in the model. Thus to produce the $V(T)$ curve, a system level model (SLM) which was more representative of a physical pyrometer system was developed. The differences between the SLM and the Monte-Carlo model are as follows:

- Filter bandpass performance is derived from manufacturer supplied data rather than ideal.

- The effects of detector size and spectral and electrical responses were derived from manufacturer supplied data rather than ideal.

- Wavelength dependent performance of glass and antireflection coating of lenses derived from manufacturer supplied data was included.

- The effects of noise on collected signals were omitted. This allows the $V(T)$ curve to be generated directly.

The SLM is similar to the first step of the Monte-Carlo model, discussed previously, which can be broken down into the following steps:

1. A blackbody curve is generated for a specified temperature, $T$.

2. The curve is then scaled to account for the collection optics.

3. Transmission through the various optics is calculated.

4. The spectrum of light reaching the detector is multiplied by the detectors' spectral response and integrated to calculate a photocurrent.

5. The electrical gain of the detector system is used to produce a voltage-temperature point.

6. The process is repeated for a range of temperatures.

Despite the increased detail of the system level model, there remain some factors that are not included, for example, errors due to chromatic aberration of the lenses and reproducibility of the filters. Due to these omissions, it is still necessary to calibrate the pyrometer to accurately interpret measurements.

The calibration was conducted as follows. First, voltages were recorded when the collection fibre was viewing the

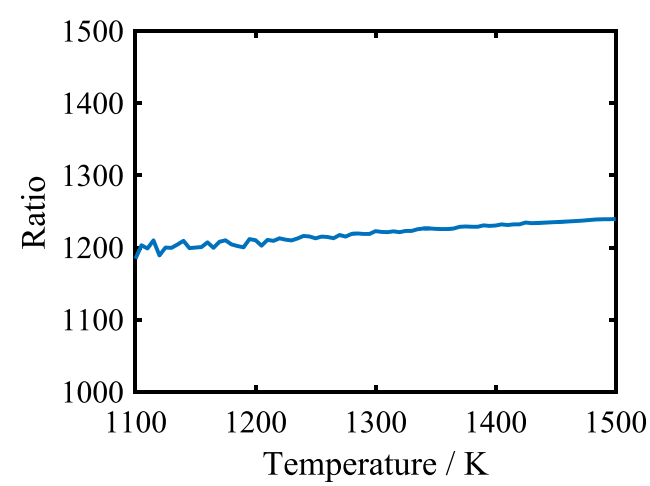

FIG. 2. A plot of the ratio of the mean output of the Monte-Carlo model to the SLM output from $1100 \mathrm{~K}$ to $1500 \mathrm{~K}$. The SLM model uses the Semrock FF01-794-32 filter, the Monte-Carlo model used an ideal filter window with a $32 \mathrm{~nm}$ passband centred at $800 \mathrm{~nm}$. 


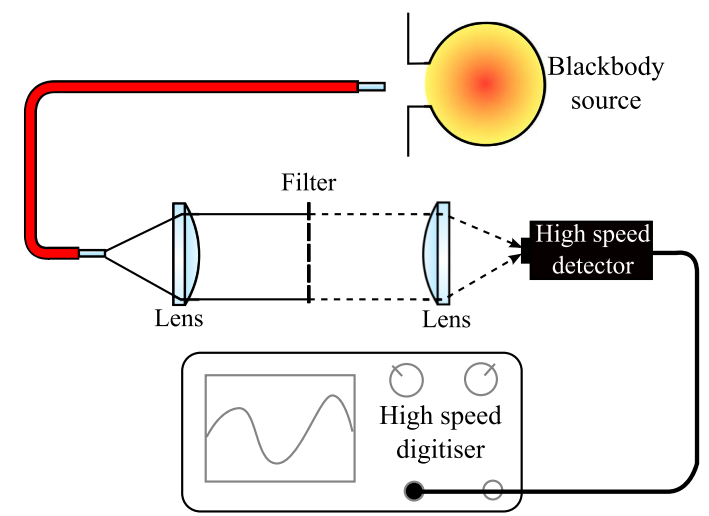

FIG. 3. Schematic diagram of the filter test setup. Light is collected from a blackbody source using an optical fibre. Light from the fibre is collimated, filtered, and then focussed onto a detector. The output from the detector is recorded on a high speed digitiser.

blackbody source at known temperatures. By comparing the voltage measurements with those predicted by the SLM, a calibration curve can be produced. This curve, together with the output from the SLM, allows voltage data to be converted directly to temperature. Following calibration it was noted that radiance levels were typically $75 \%$ of that predicted by the SLM; this discrepancy has been attributed to component imperfections and sub-optimal alignment.

The final step in validation is to relate the Monte-Carlo model to the SLM model. As discussed there are numerous factors that differ between the models. However, if the ratio of the mean output of the Monte-Carlo model to the SLM output is constant, the models would be equivalent apart from this scaling factor. Figure 2 shows the ratio of the MonteCarlo to the SLM over a range of temperatures. It can be seen that variations of up to $2 \%$ occur, indicating that the models are largely consistent but not exactly equivalent; thus the Monte-Carlo model results are comparable to the experimental results.

The most straightforward experiment that would generate data that were readily comparable to the Monte-Carlo model was measuring the effect of changing the filter passband. Three commercially available filters, of approximately equal centre frequency with varying passbands, were used for the measurements.

The experimental system used to acquire the data collected light from a blackbody source (LAND R1200P) using a $1 \mathrm{~mm}$ diameter, $0.48 \mathrm{NA}$ optical fibre (Thorlabs ${ }^{\mathrm{TM}}$ BFL481000). A lens system collected the light emitted from the fibre and directed the light, via a bandpass filter, to an amplified photodiode (FEMTO HCA-S-400M, a NEP of $68 \mathrm{pW} \mathrm{Hz}^{-\frac{1}{2}}$ was assumed), see Figure 3 . The output of the detector was recorded

TABLE I. Results from the system level model. All filters were obtained from Semrock.

\begin{tabular}{lccc}
\hline \hline $\begin{array}{l}\text { Source } \\
\text { temperature (K) }\end{array}$ & Filter & $\begin{array}{c}\text { Mean measured } \\
\text { temperature (K) }\end{array}$ & $\begin{array}{c}\text { Standard } \\
\text { deviation (K) }\end{array}$ \\
\hline 1425 & FF01-795-15 & 1425 & 39 \\
1425 & FF01-794-32 & 1423 & 17 \\
1425 & FF01-800-120 & 1420 & 5 \\
\hline \hline
\end{tabular}

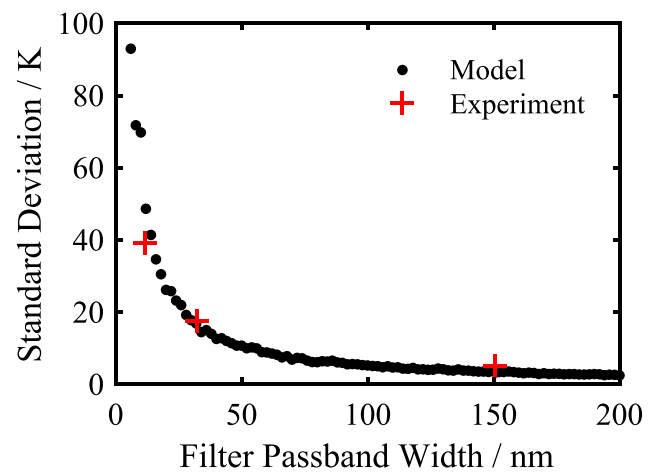

FIG. 4. A plot comparing the results from the Monte-Carlo model to data obtained experimentally by viewing a blackbody source at $1425 \mathrm{~K}$ through a spectral band.

with an 8-bit high speed digitiser (Tektronix ${ }^{\mathrm{TM}}$ DPO7254) at $1 \mathrm{GS} \mathrm{s}^{-1}$ (i.e., a bandwidth of $5 \times 10^{8} \mathrm{~Hz}$ ).

To perform the analysis, approximately 3000 points of voltage data (at each temperature) were converted to temperature using the SLM and calibration data. The mean and standard deviation of the dataset were calculated, yielding the results shown in Table I.

When using the Monte-Carlo model to calculate uncertainty, the modelled signal levels had to be adjusted to ensure appropriate noise scaling. Thus the 0.75 scaling factor determined in calibration was applied to the radiance levels in the Monte-Carlo model to account for optical inefficiencies. Experimental measurements are compared with results from the Monte-Carlo model in Figure 4. While the output of the simple model does not perfectly describe the experimental results, the fit is sufficient to validate the Monte-Carlo model for investigation of the effect of filter parameters on pyrometer performance.

\section{MONTE-CARLO MODEL RESULTS}

\section{A. $\lambda_{c}$ variation}

Following validation, the ideal model was used to investigate the effects of varying $\lambda$ and $\Delta \lambda$, assuming that the NEP of the detector was $50 \mathrm{pW} \mathrm{Hz}{ }^{-\frac{1}{2}}$; the data is recorded on an 8-bit digitiser and the blackbody radiant source was $1 \mathrm{~mm}$ in diameter with light collected from a 0.25 numerical aperture. Figure 5 shows the effect of altering the centre wavelength of the filter while maintaining a passband of $100 \mathrm{~nm}$ at temperatures of 1250,1500 , and $1750 \mathrm{~K}$. It can be seen that as the centre wavelength increases, the uncertainty decreases. Also noticeable is a transition where the uncertainty drops rapidly; this transition occurs at higher wavelengths for lower temperatures. In addition, there are diminishing returns to increasing the centre wavelength after the transition; subsequent increases in centre wavelength produce progressively smaller improvements in accuracy.

The response of the system to centre wavelength variation can be accounted for if the noise model is considered. Shifting the centre wavelength of the collection band to longer wavelengths leads to an increase in the amount of light reaching the detector resulting in a decrease in uncertainty. 


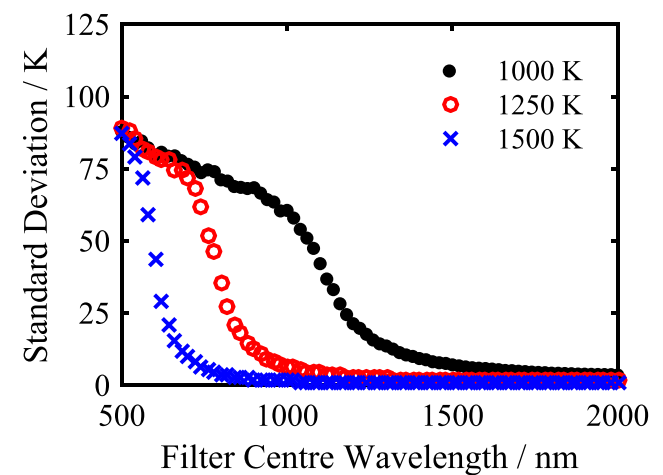

FIG. 5. A plot of calculated temperature uncertainty for a range of filter parameters. Assuming a blackbody source, centre wavelength is varied while maintaining a passband of $100 \mathrm{~nm}$.

The noise model also accounts for the transition in uncertainty as the detector noise component is constant and thus will be the dominant component at low signal levels but, once overcome, digitisation noise becomes the most significant source of noise. Digitisation noise is a fixed percentage of the signal so has a constant effect.

It is suggested that the system is configured so the centre wavelength is beyond the transition value. While a general expression for the transition value could not be determined analytically, an approach that may approximate the location of the transition is discussed in Section IV D.

\section{B. $\Delta \lambda$ variation}

Figure 6 shows the effect of altering the width of the passband of the filter while maintaining a centre wavelength of $800 \mathrm{~nm}$. Increasing the width of the passband increases the signal level which improves the accuracy of the temperature measurement. The benefit of increasing signal level due to increasing the passband continues until digitisation becomes the most significant source of noise. Again, there are diminishing returns; increases in bandpass width produce progressively smaller benefits. Changes to bandpass width produce a similar response to change to centre wavelength as the response is determined by the noise model and the same noise model was used. It can be observed that the wavelength uncertainty due to increasing the passband has a smaller effect than the benefits of increased signal level.

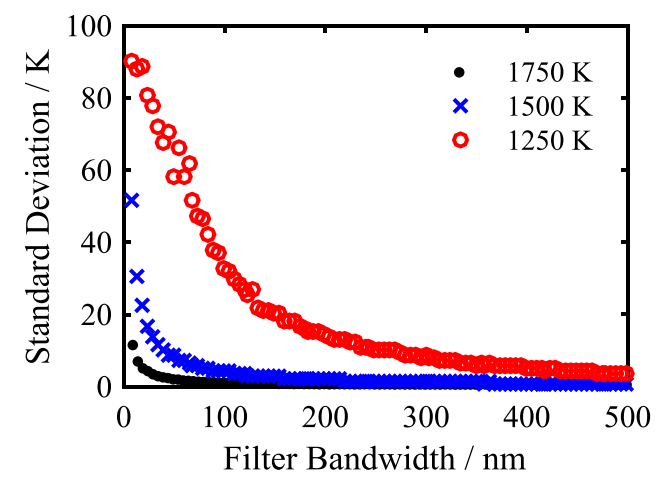

FIG. 6. A plot of calculated temperature uncertainty for a range of filter parameters. Assuming a blackbody source, passband width and temperature were varied while a centre wavelength of $800 \mathrm{~nm}$ was maintained.
Thus it appears that increasing the filter bandpass width is beneficial, however, when considering the pyrometry system as a whole, there are drawbacks. First, a wide bandpass excludes the possibility of additional bands in that spectral region. Combining multiple measurements can be beneficial, leading to a reduction in uncertainty. This is where the noise component becomes critical; if the noise scales such that a relatively large passband increase only produces small improvements in uncertainty, it maybe preferable to add another measurement band rather than continue to extend a single filter passband. Second, there are practical considerations; filters with extremely wide passbands are difficult to obtain and detectors often have limited spectral responsivity so the width of the passband cannot be increased arbitrarily.

\section{Multiple passbands}

The benefit of adding an additional measurement channel can be assessed by quantifying the effect of the change. The combination of the uncertainties from multiple measurements is given by Equation (10), where $\sigma_{\Sigma}$ is the resultant uncertainty and $\sigma_{i}$ is the $i$ th uncertainty from a set of $n$ measurements

$$
\sigma_{\Sigma}^{2}=\frac{1}{\sum_{i=1}^{n} \sigma_{i}^{-2}} .
$$

A specific example is instructive when considering multiple bands. Using Equation (10) the uncertainty from combining two measurements for filters centred at $600 \mathrm{~nm}$ and $800 \mathrm{~nm}$ viewing a $2000 \mathrm{~K}$ source with increasing passbands was calculated, Figure 7. It can seen that once the passband of the $800 \mathrm{~nm}$ filter reaches $\sim 100 \mathrm{~nm}$ there is a greater reduction in uncertainty due to adding another spectral band compared to increasing the passband to $200 \mathrm{~nm}$. Thus adding an extra measurement channel is a better use of the available spectrum.

\section{Simultaneous variation of $\lambda_{c}$ and $\Delta \lambda$}

In addition to investigating the effect of individual changes in centre wavelength and bandpass width, the idealised model was used to explore simultaneous variations in these parameters for a single passband system. If both parameters are varied, a 2D surface of uncertainty is generated. An

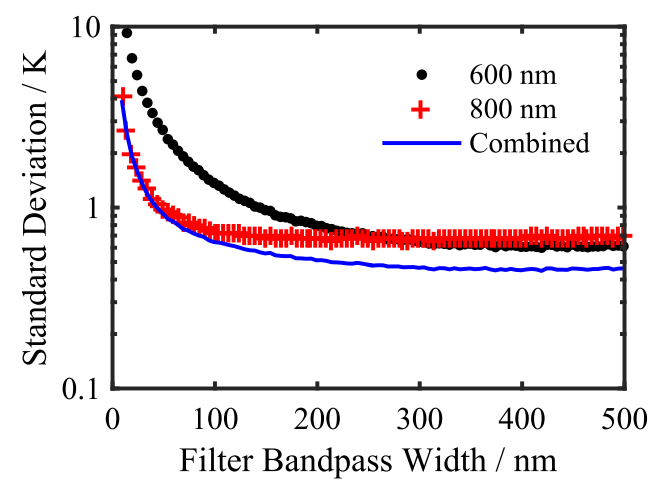

FIG. 7. A plot of the standard deviation of model results as bandpass width is varied for filters centred at $600 \mathrm{~nm}$ and $800 \mathrm{~nm}$ viewing a $2000 \mathrm{~K}$ blackbody. In addition, the standard deviation calculated when the measurements are combined is shown. 


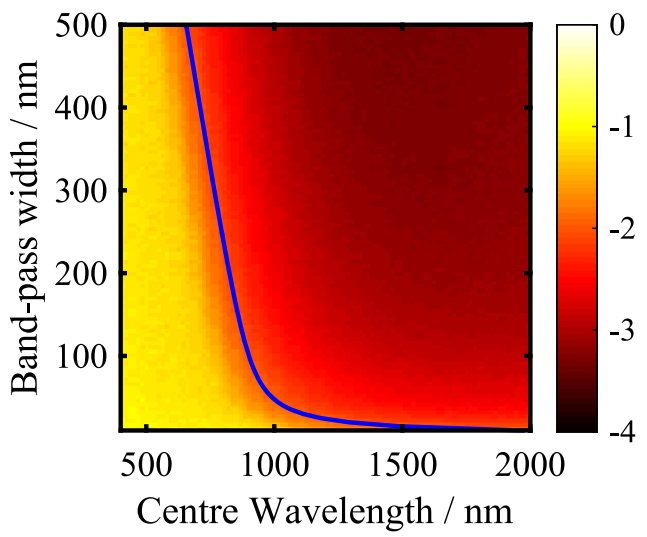

FIG. 8. A 2D image of the $\log$ of the normalised standard deviation $(\sigma / \mathrm{T})$ for a $1250 \mathrm{~K}$ blackbody source with a detector with $50 \mathrm{pW} \mathrm{Hz}^{-\frac{1}{2}}$ noise equivalent power and $500 \mathrm{MHz}$ bandwidth. A line indicating the $1 \%$ uncertainty level has been added determined by fitting the location of data points within in the range $0.9 \%$ and $1.1 \%$ with a sum of a linear function and a hyperbolic function, $f(x)=\left(\frac{a}{x}\right)^{n}$.

example of the results of this process (from a $1250 \mathrm{~K}$ source) is shown in Figure 8.

These data can be analysed to determine uncertainty "contours" where a range of centre wavelengths and bandpass widths satisfy a specified uncertainty. A $1 \%$ uncertainty contour is shown as the line on Figure 8.

A benefit of reducing the data to contour lines is that it allows for easier comparison of the effect of changing other model parameters. Figure 9 shows the $1 \%$ uncertainty contour for temperatures of 1250,1500 , and $1750 \mathrm{~K}$ with detector noise, $\sigma_{\text {det }}$, varying between 50,5 , and $0.5 \mathrm{pW} \mathrm{Hz}^{-\frac{1}{2}}$.

An empirical expression that approximates contour location has been determined. The expression specifies the centre wavelength (in $\mathrm{nm}$ ) required to attain $1 \%$ error, $\lambda_{c 1 \%}$, for a defined bandpass width, temperature, and noise level. Using variables defined earlier in this paper, the expression is as follows:

$$
\begin{aligned}
\lambda_{c 1 \%}= & -0.5 \Delta \lambda+(-0.071 \cdot \ln (\mathrm{NEP} \sqrt{B})-1.66) T \\
& +7930 \cdot(\mathrm{NEP} \sqrt{B})^{0.11} .
\end{aligned}
$$

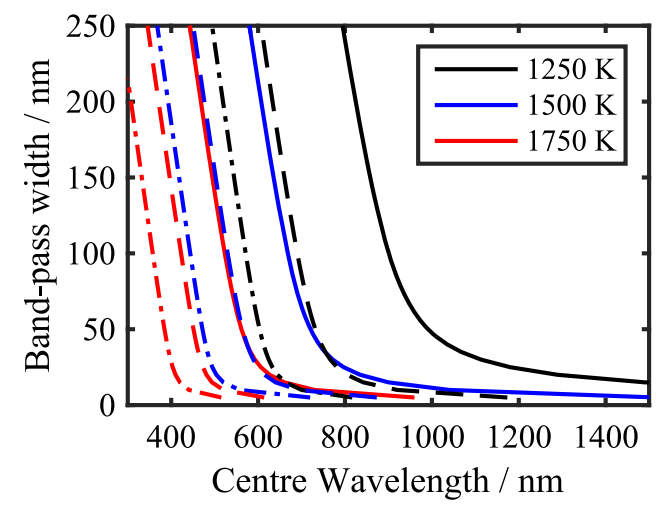

FIG. 9. Plot of uncertainty contours of $1 \%$ for blackbody source temperatures of 1250,1500 , and $1750 \mathrm{~K}$. The same uncertainty contours for the same temperatures with detector noise values of 50 (solid), 5 (dashed), and 0.5 (dot-dash) $\mathrm{pW} \mathrm{Hz}^{-\frac{1}{2}}$ are also plotted.

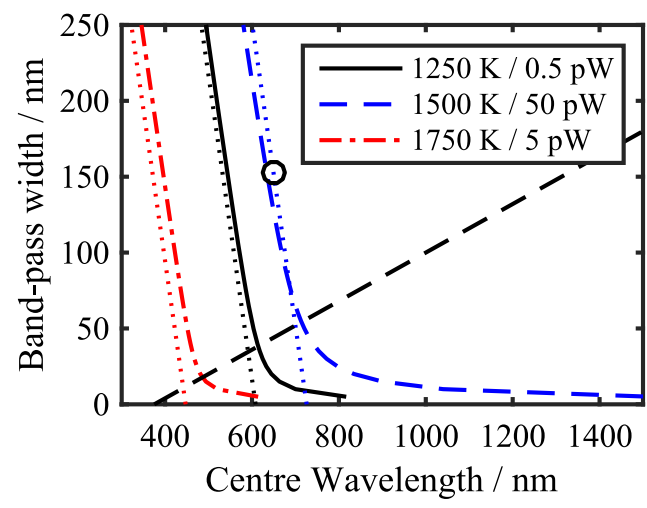

FIG. 10. Plot of contours of $1 \%$ uncertainty at selected blackbody source temperature and noise powers with associated fitted curve (dotted). The region below the dashed line indicates where results diverge from the fitting function. The circled point illustrates a solution obtained by numerically solving the fitting expression to determine the bandpass width required for a $650 \mathrm{~nm}$ centre wavelength filter.

The lines generated by the above expression are generally in good agreement with results from the simulations, however the results diverge at lower bandpass widths, see Figure 10. The lower bound (in $\mathrm{nm}$ ) for the region where Equation (11) is a good approximation for the contour locations can be approximated by the equation as follows:

$$
\Delta \lambda_{N A}<0.16 \lambda_{c}-60 .
$$

While the approximation for the 1\% contours (Equation (11)) is somewhat unwieldy to manipulate algebraically, it can be solved numerically for any of the variables (given the other parameters). For example, the expression was solved numerically to calculate the bandpass width required for a $650 \mathrm{~nm}$ centred filter with a $1500 \mathrm{~K}$ source using a $500 \mathrm{MHz}$ bandwidth detector with $50 \mathrm{pW} \mathrm{Hz}^{-\frac{1}{2}} \mathrm{NEP}$, resulting in a bandpass width of $152 \mathrm{~nm}$. This solution is displayed on Figure 10. This value is above the limit set by Equation (12) so it approximates a point on the associated simulation contour.

While Equation (11) can be used to give an indication of suitable filter parameters (for given experimental conditions) it is important to consider the limitations of the model. Two limitations are: one, the idealised model has assumed that the source is a blackbody emitter; deviation from this condition will reduce signal levels, changing the filter parameters. Two, the idealised model results considered temperatures under $2000 \mathrm{~K}$, the approximation is likely to fail if used outside this temperature range. Thus, to ensure the most appropriate filter choice, it is suggested that the insights discussed here can be used to down-select potential components for testing in a system level model that contains component level models. The system level model, together with forward modelling to estimate temperature, can then be used to optimise the configuration of the optical components.

\section{v. CONCLUSIONS}

A Monte-Carlo model has been developed and validated to investigate the performance of a pyrometry channel viewing a blackbody source while the characteristics of its associated 
filter are varied. The model was used to investigate the effect of individual variations in centre wavelength and passband width of a filter. Further investigation was conducted by considering the variation of both parameters allowing the effect of varying a noise source to be investigated.

It has been found there is a marked transition in uncertainty when varying centre wavelength. Below this transition wavelength uncertainties are large but above the transition wavelength uncertainty drops rapidly. However, increasing the centre wavelength significantly above the transition wavelength produces little reduction in uncertainty. Thus, if signal levels are high, it is usually desirable to reduce the spectral range of the filter passband and add another spectral band for measurement. The location of the transition is dependent on the filter parameters, noise levels, and the temperature that is to be measured.

Increases in filter bandpass width generally increase signal levels thus reducing uncertainty. However as the bandpass gets wider the benefit diminishes. In some cases, it may be better to add additional spectral bands for additional measurements rather than continue to increase bandpass width.

It is also observed that the increased wavelength uncertainty created by increasing the passband of filters is not noticeably detrimental to resultant uncertainty within the ranges modelled here. There is a caveat that, if the passband becomes sufficiently wide, spectral variation issues (e.g., emissivity, detector response) become significant, which would affect measurements, but these effects have not been considered in the work reported here.

Results from varying both bandpass and centre wavelength have been fitted to simplify comparison of resultant uncertainties due to changes in temperature and noise level. This has allowed an expression to be determined that approximates the bandpass width required to attain $1 \%$ uncertainty given the remaining variables (Equation (11)); this expression may be used to aid filter selection. However at lower bandpass widths, the approximation becomes inaccurate so cannot be applied; an expression describing this transition has also been determined.

\section{ACKNOWLEDGMENTS}

The authors gratefully acknowledge Imperial College London for their continued support and AWE for providing the funding for this work.

(C)Crown copyright 2017. Reproduced with the permission of the Controller of Her Majesty's Stationery Office/Queen's Printer for Scotland and AWE.

${ }^{1}$ G. A. Lyzenga, "Shock temperatures of materials: Experiments and applications to the high pressure equation of state," Ph.D. thesis, California Institute of Technology, California, 1980.

${ }^{2}$ A. Seifter, S. T. Stewart, M. R. Furlanetto, G. B. Kennedy, J. R. Payton, and A. W. Obst, "Post-shock temperature measurements of aluminium," AIP Conf. Proc. 845, 139-142 (2005).

${ }^{3}$ B. M. L. Lone, G. D. Stevens, W. D. Turley, D. B. Holtkamp, A. J. Iverson, R. S. Hixson, and L. R. Veeser, "Release path temperatures of shockcompressed tin from dynamic reflectance and radiance measurements," J. Appl. Phys. 114, 063506 (2013).

${ }^{4}$ P. B. Coates, "Multi-wavelength pyrometry," Metrologia 17, 103-109 (1981).

${ }^{5}$ D. Partouche-Sebban, D. Holtkamp, J. Pélissier, J. Taboury, and A. Rouyer, "An investigation of shock induced temperature rise and melting of bismuth using high-speed optical pyrometry," Shock Waves 11, 385-392 (2002).

${ }^{6}$ W. D. Turley, D. B. Holtkamp, L. R. Veeser, G. D. Stevens, B. R. Marshall, A. Seifter, R. B. Corrow, J. B. Stone, J. A. Young, and M. Grover, "Infrared emissivity of tin upon release of a $25 \mathrm{GPa}$ shock into a lithium fluoride window," J. Appl. Phys. 110, 103510 (2011).

${ }^{7}$ D. Partouche-Sebban, J. Pélissier, W. W. A. F. G. Abeyta, M. E. Byers, D. Dennis-Koller, J. S. Esparza, R. S. Hixson, B. J. J. D. B. Holtkamp, J. C. King, P. A. Rigg, P. Rodriguez, D. L. Shampine, J. B. Stone, D. T. Westley, S. D. Borror, and C. A. Kruschwitz, "Measurement of the shock-heated melt curve of lead using pyrometry and reflectometry," J. Appl. Phys. 97, 043521 (2005).

${ }^{8}$ A. Seifter and A. W. Obst, "About the proper wavelength for pyrometry on shock physics experiments," Int. J. Thermophys. 28, 934-946 (2007).

${ }^{9}$ T. Fu, X. Cheng, X. Fan, and J. Ding, "The analysis of optimization criteria for multi-band pyrometry," Metrologia 41, 305-313 (2004).

${ }^{10}$ M. A. Khan, C. Allemand, and T. W. Eagar, "Noncontact temperature measurement. I. Interpolation based techniques,” Rev. Sci. Instrum. 62, 392-402 (1991).

${ }^{11}$ K. Chrzanowski and M. Szulim, "Errors of temperature measurement with multiband infrared systems," Appl. Opt. 38, 1998-2006 (1999).

${ }^{12}$ W. R. Bennett, "Spectra of quantized signals," Bell Syst. Tech. J. 27, 446472 (1948).

${ }^{13}$ S. Smith, The Scientist and Engineer's Guide to Digital Signal Processing (California Technical Publishing, San Diego, CA, USA, 1997). 